\title{
Criteria for a specialist paediatric asthma clinic
}

\author{
Titus K Ninan, Dermot Ryan
}

\section{Summary}

Childhood asthma is insufficiently controlled in a large number of patients, highlighting the need for improved provision of paediatric asthma care. This paper presents consensus criteria for a paediatric asthma clinic. These are intended to provide a basis for the delivery of asthma care to children in primary and secondary care clinics.

\section{Introduction}

Asthma is a common childhood illness, imposing a major burden on society in terms of morbidity, quality of life and healthcare costs. ${ }^{1}$ The prevalence of asthma is increasing; currently an estimated 1.4 million children are treated for asthma in the UK, accounting for 1 in 8 of the paediatric population. ${ }^{2}$ Although regular use of inhaled corticosteroids may prevent the majority of asthma hospitalisations and reduce the impact of the disease ${ }^{3,4}$ the number of preschool children hospitalised for asthma is rising. ${ }^{2}$

Paediatric asthma patients are treated, diagnosed and advised on disease management in a variety of settings within primary and secondary care. However, the majority of children with asthma are managed in

Figure 1. Flow diagram of the processes involved in producing the paediatric asthma clinic criteria.

\begin{tabular}{|c|}
\hline $\begin{array}{c}\text { Core group of respiratory experts } \\
\text { Produced draft paediatric asthma clinic } \\
\text { criteria }\end{array}$ \\
\begin{tabular}{|c|} 
Respiratory expert panel \\
Appraised draft clinic criteria \\
Collated comments into clinic criteria
\end{tabular} \\
\hline $\begin{array}{c}\text { Group of respiratory experts } \\
\text { Redrafted clinic criteria to include all } \\
\text { expert views }\end{array}$ \\
\hline $\begin{array}{c}\text { Multidisciplinary consensus group } \\
\text { Discussed recommendations in draft } \\
\text { clinic criteria document }\end{array}$ \\
\hline $\begin{array}{c}\text { Final consultation process } \\
\text { Updated clinic criteria based upon } \\
\text { feedback from consensus group }\end{array}$ \\
\hline $\begin{array}{c}\text { Criteria required for a paediatric } \\
\text { asthma clinic }\end{array}$ \\
Final version of clinic criteria \\
\hline
\end{tabular}

Core group of respiratory experts (panel 1)

Produced draft paediatric asthma clinic criteria document primary care asthma clinics.

Although there is currently

no formal accredited asthma training for general

practitioners (GPs) in

primary care in the UK,

most staff in such clinics

have some level of asthma

management training. In

recent years the number of

nurse-led asthma clinics in

general practice has

increased, providing a useful approach to the management of childhood asthma. ${ }^{5,6}$ It is

likely that the near future

will see the establishment of GPs with a specialist clinical interest (GPwSI) in respiratory disease, participating in the management of children with asthma. 7

The quality of asthma care is dependent on establishing the correct diagnosis for asthma, selecting appropriate treatment, instructing disease management techniques and developing a partnership between care provider and patient to promote adherence to treatment plans.

The British Thoracic Society
(BTS) and the Scottish Intercollegiate Guidelines Network (SIGN) guidelines on asthma management cover the diagnosis and the long-term and acute management of asthma in adults and children. ${ }^{8}$ National asthma guidelines have been influential in improving the diagnosis and management of asthma in children, but many children still suffer frequent symptoms.

The recent Asthma Insights and Reality in Europe (AIRE) survey showed that paediatric asthma management and control were suboptimal in all of the seven Western European countries surveyed. ${ }^{9}$ A community-based study of children with asthma has demonstrated that many children experience inadequate control of asthma symptoms, reflecting the suboptimal provision of paediatric asthma care in the UK.10 Further efforts to fully implement asthma guidelines are therefore required to improve asthma control in children. ${ }^{9,11}$

The need to improve paediatric asthma care has motivated a coalition of experts in respiratory medicine to collaborate in developing minimum criteria for a paediatric asthma clinic. These criteria are intended to support best practice and should be used in conjunction with current guidelines for asthma management. ${ }^{8}$ It is intended that these criteria should be implemented in primary and secondary care asthma clinics where children are seen, rather than in those services that provide care for acute asthma episodes. Ultimately, the criteria aim to drive improvements in standards of care provided to children and support the integration of primary and secondary care management options, thus providing consistency of care.

Consensus-based approach towards criteria for a specialised paediatric asthma clinic

The essential asthma clinic criteria were developed using a consensus-based approach, involving expert opinion from those actively involved in developing clinical practice (Figure 1). The clinic criteria presented in this article were submitted to many healthcare professionals, from a variety of disciplines, for their endorsement and support. This was an active process and their suggestions are reflected in the final version of these clinic criteria.

\section{Overview of the clinic criteria}

The clinic criteria have been specifically designed to support integrated care pathways and to promote 


\section{Table 1. Full list of criteria for a specialist asthma clinic where children are seen}

\section{Services for Patients}

Criteria for primary and secondary care: Patients are able to obtain the services of the team at flexible times and without delay

Structure - essential requirements

\section{Criteria}

Doctors with experience and training in paediatric asthma

Nurses with experience and training in paediatric asthma

\section{Secondary care}

New entrants: 1-year paediatric respiratory

training and completion of approved qualifications

Existing clinicians: substantial experience in the provision of a quality service for asthma patients All: evidence of ongoing professional development Note: a lead clinician with specialist training is desirable in this setting

For most Trusts, a paediatric nursing qualification is mandatory

RSCN/RNCB qualification and accredited respiratory diploma as an additional requirement Ongoing mentoring and monitoring

Completion of a post-registration accredited-level 2 paediatric respiratory module and ongoing training and competency

Facilities and trained personnel to measure and interpret lung function tests in children over 5 years of age

\section{Access to radiology diagnostic services \\ A child-friendly environment \\ Provision for procedures in structure of care, including audit}

Spirometer with printout and flow volume loops

Staff trained to use a spirometer

Equipment to be callibrated according to manufacturers standards

Access to appropriate investigations, eg. endoscopy and imaging and young people

Evidence of periodic audit
Safe environment that ensures parental participation and allows privacy and dignity for older children

Age-appropriate toys, games and educational materials

Trained personnel and callibrated equipment to measure height and weight Educational, training, demonstration and monitoring materials and equipment

A register of asthma patients

\section{Structure - desirable requirement}

Access to allergy and additional respiratory

diagnostic services

Educational, training, demonstration and

monitoring materials and equipment

\section{Process - essential requirements}

\section{Criteria}

System of appointments and structured recall
Secondary care

Flexible appointment system DNA policy
Primary care

Evidence of experience in the management of paediatric asthma

Evidence of ongoing training in the management of asthma

Note: there is currently no accredited asthma training for GPs in in primary care

Appropriate paediatric experience

Accredited-level asthma training*

Ongoing mentoring and monitoring

Completion of a post-registration accredited level-2 paediatric respiratory module and ongoing training and competency

*Note: a lead nurse should possess a diploma in asthma care

Lung function equipment - peak expiratory flow meter*

Protocol for referral for more sophisticated tests

Note: ensure single-use mouthpieces

Access to appropriate investigations eg. endoscopy \& imaging Facilities to ensure patients are informed of their results
Height and weight should be measured using regularly maintained and callibrated equipment, and the results recorded in current centile charts

Appropriate range of devices, peak flow, symptom diaries and personal management plans Provision of educational tools with access to multi-lingual translation where appropriate

Available across primary and secondary care, especially in Accident and Emergency (A\&E) departments To include "at risk" patients as defined by the BTS/SIGN 2003 guidelines ${ }^{8}$
Access to allergy testing and other laboratory tests carried out by trained staff

\section{Appropriate referral}

Use of spirometer by trained staff

Equipment callibrated according to manufacturers standards

\section{Primary care}

Flexible appointment recall system

DNA policy

Guidelines for emergency appointments and recal

A review policy in line with asthma guidelines

Targeted review of medication

Guidelines and facilities to treat-stabilis and follow-up acute episodes of asthma

Access to oxygen, spacer device/metered-dose Access to oxygen, spacer device/metered-dose inhaler, oral steroids and nebuliser and resuscitation equipment

Follow BTS/SIGN guidelines or locally agreed guidelines underpinned by the BTS/SIGN Guidelines

Effective community, primary and secondary care interface

Explicitly agreed channels of communication across primary and secondary care

Written guidelines for referral to secondary care

Regular and timely communication after attendance or admission at A\&E and outpatient departments

Agreed policy in practice for when a patient should be seen urgently and as part of a structured care plan

Parents and guardians to be included in all correspondence

Regular team discussion regarding difficult-to-manage asthma patients

This should include a multi-professional/multi-agency approach

System for identifying at-risk patients, and a register of these patients

Note: factors for identifying at risk patients include: previous asthma, lack of attendance, overuse of ß2-agonists, nebuliser use, lack of parental supervision, recent A\&E visit and other comorbidity factors

Individual written management plans Plans following National Asthma Campaign template or similar

Height and weight plotted on centile chart

Process - desirable requirements

Criteria

Plot height and weight at each review on up-to-date charts

Guidelines and facilities to treat/stabilise

and follow up acute episodes of asthma

Clinic able to participate in research activities

Outcome

Criteria

Secondary Care

Primary Care

Access to paediatric oximeter

\section{Database. ongoing research programme}

Secondary care

Primary care

Programme of audit meeting

as part of clinical governance 
Figure 2. Summary of criteria for a specialised paediatric clinic.

Services for patients - primary care / secondary care Patients are able to obtain the services of the team at flexible times and without delay.

\section{Structure - essential requirements}

1. Doctors with experience and training in paediatric asthma (implementing this out of hours may be problematic)

2. Nurses with experience and training in paediatric asthma. (implementing this out of hours may be problematic)

3. Facilities and trained personnel to measure and interpret lung function tests in children over 5 years of age.

4. Access to radiology diagnostic services.

5. A child-friendly environment.

6. Provision for procedures in structure of care, including audit.

7. Trained personnel and calibrated equipment to measure height and weight.

8. Educational, training, demonstration and monitoring materials and equipment.

9. A register of asthma patients.

Structure - desirable requirements

10. Access to allergy and additional respiratory diagnostic services.

11. Educational, training, demonstration and monitoring materials and equipment.

Process - essential requirements

12. System of appointments and structured recall. Adequate information technology infrastructure.

13. Guidelines and facilities to treat/stabilise and follow up acute episodes of asthma.

14. Effective community, primary and secondary care interface.

15. Regular team discussion regarding difficult-to-manage asthma patients.

16. Individual written management plans.

17. Height and weight plotted on a centile chart.

Process - desirable requirements

18. Guidelines and facilities to treat/stabilise and follow up acute episodes of asthma.

19 Clinic able to participate in research activities.

\section{Outcome}

20. Ongoing audit process by primary care team as part of clinical governance. consistency in this difficult area of clinical practice. To enable their implementation, it is essential that patients should be able to obtain the services of primary or secondary care teams at flexible times and without delay. The clinic criteria are defined under three different categories, encompassing structure, process and outcome, and are shown in full in Table 1 and summarised in Figure 2. The criteria are intended to support standards of care delivery and are divided into essential and desirable requirements for running a specialist asthma clinic where children are seen. Primary and secondary care clinics are considered separately where appropriate, but, depending on the size of the clinic,

intermediate criteria between primary and secondary care may be applied. These criteria are designed to complement current guidelines for the management of asthma in children. ${ }^{8}$

\section{Conclusions}

The prevalence of childhood asthma dictates the importance of effective intervention in both primary and secondary care clinics. Careful consideration is needed to ensure that such clinics provide appropriate interventions in terms of both therapies and provision of information for patients.

Efforts at national level towards the implementation of these clinic criteria for a specialist paediatric asthma clinic will only have an impact on asthma care if the information contained within them is widely disseminated and resources identified to ensure their implementation. It is imperative that this information can be accessed by both those commissioning care and by care providers alike. Continued involvement at local and national level is necessary to ensure that the message reaches those who deal with children with asthma on a regular basis leading to the evolution of a consistently high standard of service provision.

In assessing the effective delivery of asthma care it is important to use appropriate validated outcome measures that are applicable to both the primary and secondary care setting. The national asthma guidelines provide outcome measures for primary and secondary hospital clinics to assist in auditing the quality of asthma care given. ${ }^{8}$

These clinic criteria provide a framework for an organised process of care provision in paediatric primary and secondary asthma clinics, leading to a standardised level of care for all patients. Any processes should include a seamless transition between care in primary and secondary services. ${ }^{12}$

\section{Acknowledgements}

The authors would like to thank 3M Health Care for financial support from an unrestricted educational grant and Complete Medical Communications Ltd for facilitating the development of the clinic criteria. The following UK-based healthcare professionals contributed to the development of the clinic criteria: Dr Neil Barnes, The London Chest Hospital, London; Professor Steve Chapman, Keele University, Keele; Professor Paul Corris, Freeman Hospital, Newcastle upon Tyne;

Dr Jonathan Couriel, Royal Liverpool Children's Hospital, Liverpool;

Dr Kevin Gruffydd-Jones, Box Surgery, Box;

Dr John Haughney, University of Aberdeen, Aberdeen; Dr Rupert Jones, Roborough Surgery, Plymouth;

Ms Sarah Latham, King's College Hospital, London;

Ms Melinda Letts, Commission for Health

Improvement, London;

Dr Mark Levy, Kenton Bridge Medical Centre,

Harrow;

Dr Vincent McGovern, Ochilbeg, Belfast;

Dr Spencer Nicholson, Salford Primary Care Trust,

Salford;

Professor David Price, University of Aberdeen, Aberdeen;

Ms June Roberts, Langworthy Medical Practice,

Salford;

Dr Dermot Ryan, Woodbrook Medical Centre,

Loughborough;

Dr Paul Seddon, Royal Alexandra Hospital for Sick

Children, Brighton;

Ms Judith Smith, Barn Surgery, Gillingham;

Dr Alan Smyth, Nottingham City Hospital,

Nottingham;

Dr Jeffrie Strang, North Yorkshire Health Authority, York;

Dr Huw Thomas, Bristol Royal Hospital for Children, Bristol;

Dr Mike Thomas, Minchampton Surgery, Stroud;

Ms Esther Threfal, British Lung Foundation, London; Mrs Trisha Weller, National Respiratory Training 
Original research

\section{For personal use only.}

\section{Not to be reproduced without the permission of the Primary Care Respiratory Journal}

Centre, Warwick;

Ms Edwina Wooler, Royal Alexandra Hospital for Sick Children, Brighton.

\section{References}

1. Lenney W. The burden of pediatric asthma. Pediatr Pulmonol Suppl 1997; 15:13-16.

2. National Asthma Campaign. Out in the open: a true picture of asthma in the United Kingdom today. Asthma J 2001; 6:3-14

3. Audit Commission. A prescription for improvement: towards more rational prescribing in general practice. In: London: HMSO, 1994.

4. Suissa S, Ernst P, Kezouh A. Regular use of inhaled corticosteroids and the long term prevention of hospitalisation for asthma. Thorax 2002; 57:880-4. 5. Dickinson J, Hutton S, Atkin A, Jones K. Reducing asthma morbidity in the community: the effect of a targeted nurse-run asthma clinic in an English general practice. Respir Med 1997; 91:634-40.

6. Dickinson J, Hutton S, Atkin A. Implementing the British Thoracic Society's guidelines: the effect of a nurse-run asthma clinic on prescribed treatment in an English general practice. Respir Med 1998; 92:264-7.
7. Williams S, Ryan D, Price D, et al. General practitioners with a special clinical interest: a model for improving respiratory disease management. $\mathrm{BrJ}$ Gen Pract 2002; 52:838-43.

8. British guideline of the management of asthma. A national clinical guideline. The British Thoracic Society/Scottish Intercollegiate Guidelines Network Thorax 2003; 58(suppl1):1-94.

9. Vermeire PA, Rabe KF, Soriano JB, Maier WC. Asthma control and differences in management practices across seven European countries. Respir Med 2002; 96:142-9.

10. Price D, Ryan D, Pearce L, et al. The burden of paediatric asthma is higher than health professionals think: results from the Asthma In Real Life (AIR) study. Prim Care Respir J 2002; 11:30-3.

11. Rabe KF, Vermeire PA, Soriano JB, Maier WC. Clinical management of asthma in 1999: the Asthma Insights and Reality in Europe (AIRE) study. Eur Respir J 2000; 16:802-7.

12.Neville RG, Higgins BG. Providing better asthma care: what is there left to do? Thorax 1999; 54:813-7.
Titus Ninan

Consultant Paediatrician

\section{Dermot Ryan}

General Practitioner

Correspondence to:

Dr Titus Ninan

Birmingham Heartlands

Hospital

Bordesley Green East

Birmingham

B9 5SS

$\mathrm{Tel}+44(0) 1214243823$

Fax $+44(0) 1214240827$

Email:

titus.ninan@heartsol.wmi ds.nhs.uk

Date submitted: 08/12/02

Date Accepted: 05/05/03

Prim Care Resp J 2003;

12(2):42-45 